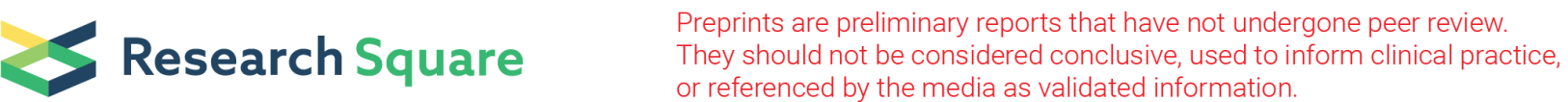

\section{Learning Curves of Robotic Spine Surgery in a System Lack of Active Perception: Potential Roles of Teamwork and Unmet Needs}

\section{Yu-feng Su}

Kaohsiung Medical University Chung-Ho Memorial Hospital

Tai-Hsin Tsai

Kaohsiung Medical University Chung-Ho Memorial Hospital

Keng-Liang Kuo

Kaohsiung Medical University Chung-Ho Memorial Hospital

Chieh-Hsin Wu

Kaohsiung Medical University Chung-Ho Memorial Hospital

Cheng-Yu Tsai

Kaohsiung Medical University Chung-Ho Memorial Hospital

Yen-Mou Lu

Kaohsiung Medical University Chung-Ho Memorial Hospital

Shiuh-Lin Hwang

Chi-Hsien Spine Hospital, Kaohsiung, Taiwan

Pei-Chen Lin

Kaohsiung Medical University

Ann-Shung Lieu

Kaohsiung Medical University Chung-Ho Memorial Hospital

Chih-Lung Lin

Kaohsiung Medical University Chung-Ho Memorial Hospital

Chih-Hui Chang ( $\nabla$ chchang20@gmail.com)

Kaohsiung Medical University Chung-Ho Memorial Hospital

\section{Research Article}

Keywords: Robotic spine surgery, learning curve, human computer communication, teamwork, humanrobot collaboration

Posted Date: December 7th, 2021

DOI: https://doi.org/10.21203/rs.3.rs-1122747/v1 
License: (c) (i) This work is licensed under a Creative Commons Attribution 4.0 International License. Read Full License 


\section{Abstract}

Background: The aim of this study was to investigate the learning curve of robotic spine surgery quantitatively with the well-described power law of practice.

Methods: Kaohsiung Medical University Hospital set up a robotic spine surgery team by the neurosurgery department in 2013 and the orthopedic department joined the well-established team in 2014. A total of 150 cases and 841 transpedicular screws were enrolled into 3 groups: the first 50 cases performed by neurosurgeons, the first 50 cases by orthopedic surgeons, and 50 cases by neurosurgeons after the orthopedic surgeons joined the team. The time per screw and accuracy by each group and individual surgeon were analyzed.

Results: The time per screw for each group was $9.56 \pm 4.19,7.29 \pm 3.64$, and $8.74 \pm 5.77$ minutes respectively. The accuracy was $99.6 \%$ (253/254), 99.5\% (361/363), and 99.1\% (222/224), respectively. The first group took significantly more time per screw, but without significance on the nonlinear parallelism test.

Analysis of 5 surgeons and their first 10 cases of short segment surgery showed the time per screw by each surgeon was $12.28 \pm 5.21,6.38 \pm 1.54,8.68 \pm 3.10,6.33 \pm 1.90$, and $6.73 \pm 1.81$ minutes. The first surgeon who initiated the robotic spine surgery took significantly more time per screw and the nonlinear parallelism test also revealed only the first surgeon had a steeper learning curve.

Conclusions: This is the first study to demonstrate that differences of learning curves between individual surgeons and teams. The roles of teamwork and the unmet needs due to lack of active perception are discussed.

\section{Introduction}

Robotic-assisted technologies have been introduced into the practice of spinal surgery, mainly focusing on transpedicular screw placement over recent years. The accuracy of robot-assisted pedicle screw placement has been a focus of research (1). However, few studies address the learning curve of robotic spine surgery $(2-11)$.

The teamwork, including human-robot team interaction, workplace culture, and experiences of surgeons can affect the processes and outcomes of robotic endoscopic surgery.(12) Interestingly, the relationship between teamwork and the learning curve has rarely been mentioned in the field of robotic spine surgery. Furthermore, active perception, one of key components in robotics, has never been mentioned in any study or research protocol of spinal robotic surgery.

Kaohsiung Medical University Hospital (KMUH) set up the robotic spine surgery team in May 2013. Up until July 2017, the members of the team had performed 688 cases and implanted 3,896 transpedicular screws. The orthopedics department joined the robotic spine surgery team in August 2014. At that time, 
255 cases had been carried out and 1237 screws had been placed successfully by the well-established team, mainly cooperating with the department of neurosurgery (Figure 1). The perioperative teamwork of this team has been emphasized especially by active communication and the "triple-check process".

This study aimed to investigate the learning curve of the robotic spine surgery via analyzing the accuracy and the surgical time of transpedicular screw placement. Case series with specific time sequences were collected for analysis to clarify the possible correlation among team, teamwork, individual surgeons, and learning curves.

\section{Materials And Methods}

\section{Ethics statement}

This clinical study was approved by the Institutional Review Board of Kaohsiung Medical University Hospital (No: KMUHIRB-E(I)-20150167). All methods were performed in accordance with the relevant guidelines and regulations. Written informed consent was obtained from all participants. Prior to analysis, patient data were de-identified and anonymously analyzed.

\section{Patient selection}

Between May 2013 and June 2017, there were 688 patients consecutive who received thoracolumbar surgery using the Renaissance robotic system (Renaissance ${ }^{\mathrm{TM}}$; Mazor Robotics Ltd., Caesarea, Israel) at $\mathrm{KMUH}$. A total of 3,896 screws were implanted successfully. We retrospectively analyzed all the patients. Cases of spinal malignancy or spinal infection were excluded from the study. Three groups of patients were enrolled and analyzed:

(1) Neurosurgeon (NS) early 50: The first 50 cases performed by the department of neurosurgery and the robotic spine surgery team between May 2013 and July 2013.

(2) Orthopedic early 50: The first 50 cases performed by the department of orthopedics and the robotic spine surgery team between August 2014 and September 2015.

(3) NS later 50: 50 consecutive cases performed by the department of neurosurgery and the robotic spine surgery team between August 2014 and November 2014 (just after the department of orthopedics joined the team).

The characteristics of patients in the 3 groups were reviewed and analyzed (Table1). The time per screw, assessed as the learning curve of the team, was calculated and plotted respectively (Figure 2).

Separately, the individual learning curves of surgeons were investigated with the short-segment surgery (2 segments, 4 pedicle screws). All the surgeons in this study are experienced spine surgeons with high surgical volume (up to 300 cases/year). The first 10 cases with a $100 \%$ accuracy for 5 individual 
surgeons (50 cases total, 40 screws total per surgeon) were enrolled for analysis. The time per screw was measured and plotted (Table 2 and Figure 3 ).

\section{Robotic spine surgery techniques}

The Renaissance robot-guided system has been described in previous studies $(13,14)$. Five main steps were carried out to place transpedicular screws with the Renaissance robot-guided system:

1. Preoperative planning: spiral computed tomography (CT) scanning (1-mm intervals) of the spine was performed preoperatively to reconstruct the 3D plane of the spine and to select the optimal screw placement strategy.

2. Mounting: the mounting system was attached to an appropriate bony structure of the spine to maintain stability during registration. The robotic arm of the guided system was also fixed to the bony structure to provide intraoperative guidance.

3. Registration: the Renaissance robot-guided system automatically registered the intraoperative images with the preoperative CT images by comparing anteroposterior and oblique views of radiographic images.

4. Drilling: the robot was attached to a mounting frame and moved to the position selected during preoperative planning. After directing the guiding tube to the pedicle, the surgeon performed the drilling. The K-wire was then placed with robotic guidance. During the process of drilling, there is no passive nor active perception processs/mechanims provided by the robotic system.

5. Intraoperative accuracy evaluation: after the K-wire implantation was completed, anteroposterior and lateral fluoroscopic images were obtained. The accuracy was evaluated and repositioning of the Kwire was achieved manually in cases where the accuracy was not acceptable. Secondary registration, developed by the team at $\mathrm{KMUH}$, with anteroposterior and oblique fluoroscopic images and the Renaissance robot-guided system, was carried out in cases after January $2015(13,14)$. Reposition of the K-wire was done again with the robotic system. Manual reposition was only applied when the reposition was still unacceptable.

\section{Team building and teamwork}

\section{Team building}

The robotic spine surgery team at KMUH is made up of qualified surgeons ( 7 neurosurgeons and 3 orthopedic surgeons), coordinators, radiological technicians, specialist nurses, surgical technicians, and surgical nursing staff. Surgeons and surgical technicians are all qualified in the Renaissance robotics system training programs, including cadaver workshop. The coordinators and specialist nurses manage the preoperative preparation and perioperative care. The parameters and image quality of CT scans are examined carefully by radiological technicians and surgeons. 
The members meet the day prior to surgery to discuss the surgical plan. The process and possible factors influencing the placement of K-wires (14) are previewed and examined. Five main steps mentioned above are carried out to place transpedicular screws with the Renaissance robot-guided system. The "triplecheck process" is applied during the surgery, i.e. pointing, reading, and confirming all parameters of the settings and instruments with team members involved in every step. Pointing means the members should point with fingers the parameters shown on the screen of robot. Reading means the members should read together the parameters they point. Confirming means the member agree the parameters they point and read. The process and results are recorded immediately following every procedure.

\section{Workplace culture}

The Joint Commission International's ( $\mathrm{JCl}$ ) accreditation standards for hospitals (Joint Commission International, Oak Brook, Illinois, USA) are followed by the team perioperatively, as KMUH has partnered with the $\mathrm{JCl}$. Patient-safety Reporting system has been setup and staffs are encouraged to communicate actively and report possible adverse events with name or anonymously.

\section{Statistical analysis}

The Chi-squared test and Wilcoxon sign rank test were applied for categorical and continuous variables. Mean values are presented as mean \pm standard deviation (SD). The learning curves (team and individual) were fit and plotted on a nonlinear platform. The following equation was used:

$$
\frac{\mathrm{c}}{[1+\operatorname{Exp}[-a *[\text { serial }-b]]]}
$$

Where $a=$ growth rate, $b=$ inflection point, and $c=$ asymptote.

The parallelism of learning curves was measured and tested with the parallelism $\mathrm{F}$ test. All statistical analyses were performed using the JMP 12 software (SAS Institute Inc., Buckinghamshire, UK). The level of significance was set at 0.05 .

\section{Results}

\section{The learning curves of the team and departments}

Table 1 shows the characteristics of the 3 groups. There were no significant differences among the basic characters of patients. The orthopedic early group performed more long segments cases ( $\geqq 3$ levels and $\geqq 6$ screws). The total 150 cases and 841 screws in the 3 groups had a time per screw of $9.56 \pm 4.19$, $7.29 \pm 3.64$, and $8.74 \pm 5.77$ minutes for the NS early, orthopedic early, and NS later groups, respectively. The NS early group took significantly more time per screw $(p=0.0017)$, but this was not significant after the nonlinear parallelism test (growth rate estimate was $-0.01 \pm 0.01,-0.05 \pm 0.17$, and $-0.01 \pm 1.25, p=0.85$, Figure 1). The accuracy for each group was $99.6 \%$ (253/254) for NS early, $99.5 \%$ (361/363) for 
orthopedic early, and $99.1 \%$ (222/224) for NS later. There was no significant difference in the accuracy between the 3 groups.

\section{The individual learning curves of surgeons}

Five surgeons (4 neurosurgeons and 1 orthopedic surgeon) and their first 10 cases of short segment surgery ( 2 levels and 4 screws) were enrolled for the analysis of individual learning curves (Table 2 ). The time per screw of each surgeon was $12.28 \pm 5.21,6.38 \pm 1.54,8.68 \pm 3.10,6.33 \pm 1.90$, and $6.73 \pm 1.81$ minutes. The first surgeon who initiated the robotic spine surgery in the team took significantly more time per screw $(p=0.001)$, and the nonlinear parallelism test (Figure 2$)$ also showed that only the first surgeon had a steeper learning curve (growth rate estimate was $-0.13 \pm 0.17,0.08 \pm 0.92,-1.41 \pm 0.0,-0.57 \pm 1.6$, $0.75 \pm 0.0, p<0.0001)$.

\section{Discussion}

\section{The learning curves between team and individual surgeons}

This study demonstrates the learning curve of both the team as a whole as well as individual surgeons. With an established-team and standardized teamwork, the learning curve of a newly joined but qualified member of the surgical team may be parallel with the other experienced members in the team. To the best of our knowledge, this could be the first study to show the learning curves of robotic spine surgery with quantificational and nonlinear analysis.

The power law of practice is well described quantitatively for human learning curve study in psychology (15). This law states that the logarithm of the reaction time for a particular task decreases linearly with the logarithm of the number of practices. Therefore, the time per screw is viewed as an important element of the learning curve during robotic spine surgery $(4,8)$. Our results, as the first study, demonstrated a similar power law of practice only on the learning curve of the first surgeon, who initiated the robotic spine surgery for the team. In the series by Urakov et al. (8), the initial academic experiences of residents/fellows and their learning curve with robotic spine instrumentation were explored. No significance was noted regarding the speed of pedicle instrumentation under senior surgeons' guidance. According to the theory, results and observations, a well-established team, teamwork, and supervision are important factors for the learning curve of robotic spine surgery.

However, the characteristics of the smooth power law may potentially mask more complex dynamics underpinning individual learning curves. Therefore, using a single power law to predict or analyze individual performance may obscure more complex learning dynamics (16).

\section{The accuracy of robotic spine surgery}

The accuracy of spinal instrumentation is another important issue in robotic spine surgery. Previous studies have shown that the rate of successful robotic-assisted pedicle screw placement became consistent after 20 or 30 cases $(6,10)$. In our study, the rate of accuracy did not change significantly 
between the 3 groups of 50 cases during 3 specific time intervals. In a meta-analysis of robotic spine surgery by Joseph et al. (17), including 22 retrospective case series and prospective randomized trials, the consistency and high accuracy rates of robotic spine surgery were also recognized. Ringel et al. (3) also stated that accuracy did not improve through the course in their study. Obesity, osteoporosis, and congenital scoliosis have been recognized as risk factors for screw malposition and surgeons in the initial stage of using a robot are suggested to avoid performing surgery on patients with these risk factors (1).

One of our previous studies developed a secondary registration protocol that increased the success rate and intraoperative accuracy by the same robotic system (13). In the studies we published in 2016 and $2017(13,14)$ showed that the K-wire needed to be repositioned manually is $1.26 \%(4 / 317 \mathrm{~K}$-wires, with secondary registration) and $0.15 \%$ (1/662 K-wires, with third registration). Factors influencing accuracy can be errors in preoperative planning, mounting, registration, drilling, or robot assembly (14). All of these factors could be eliminated or minimized by a well-established team and teamwork, according to the results of this study.

\section{Potential roles of teamwork}

Effective teamwork can be measured by examining the quality of output, the process and the members' performance (18). Team dynamics are important for efficient teamwork. Team dynamics include open communication to avoid conflicts, effective coordination to avoid confusion, efficient cooperation to perform the tasks in a timely manner and produce the required results, and high levels of interdependence to maintain high levels of trust, risk-taking, and performance (19). The smooth and parallel learning curves of the team and individuals imply the potential roles of teamwork in robotic spine surgery.

Communication and workplace culture are thought to be important factors in the human-robot team interaction (12), but the evaluation and measurements are usually difficult in complex operation rooms. In the surgery with the Renaissance robot-guided system, a potential error could take place during registration due to inconsistencies between the numbers of station number set on the computer and on the mounting platform. The "triple-check process" applied in robotic spine surgery, i.e. pointing, reading, and confirming all parameters of settings and instruments with team members in every step, prevents our team from errors. There is little literature related with human-robot interaction in the field of robotic spine surgery. Though we did not propose new experimental study design about the human-robot interaction in this study, the high accuracy of transpedicular screw implantation in this study demonstrates not only the efficiency of surgical robot but also the efficiency of the good human-robot interaction.

KMUH has been an academic medical center in southern Taiwan since the 1970s. It has received accreditation every three to five years by the official accreditation organizations in Taiwan. Furthermore, the partnership with the international accreditation organization also encourages and regulates the team to follow rules and guidelines more strictly. 
We believed this is the first article emphasizing the importance of team building, human-robot interaction and workplace culture in robotic spine surgery.

\section{Robotic spine Surgery - a system lack of active perception and unmet needs}

Modeling and control strategies for perception, defining active perception (20), are missing in the robotic system we used for this study. According to our previous study, skiving over a steep slope of bony surface is the main factor affecting accuracy (14). However, during the process of drilling, there is no passive nor active perception process or mechanism provided. The members of surgical team use their naked eyes and fingers to detect possible skiving of guiding tube before, during, and after the drilling procedure. Contrary to intraoperative image-guided spinal navigation, there is no guiding image or visual feedback provided by the robotic system during the drilling procedure and screw placement (21). These defects are also barriers to surgeons to use or trust this robotic system. Intelligent control strategies according to the data from detecting possible deviation of guiding tube, are apparently the unmet needs for robotic spine surgery.

\section{Conclusion}

With teamwork, the learning curve of robotic spine surgery for a newly employed surgeon can be smooth and parallel with other experienced surgeons. The accuracy is also high and consistent. Communication and workplace culture are important for teamwork meanwhile triple-check process is advocated during robotic spine surgery. We propose active perception is the unmet need for current robotic spine surgery.

\section{Declarations}

\section{Consent for publication}

No images, personal or clinical details of participants are presented that compromise anonymity

\section{Conflict of Interest}

The authors declare that the research was conducted in the absence of any commercial or financial relationships that could be construed as a potential conflict of interest.

\section{Author Contributions}

Conceived and designed the study: YFS, CHC, THT, CLL

Performed the study and Analyzed the data: YFS, PCL

Contributed materials/analysis tools: YFS, CHC, KLK, CHW, CYT, YML, SLH, ASL, CLL

Wrote the paper: YFS, CHC, THT, CLL 
All authors have read and approved the manuscript.

\section{Acknowledgments}

Thanks to all the staff of Robotic Spine Surgery Team and Department of Neurosurgery at KMUH. This work was supported by part of grants KMUH104-4M20 and KMUH105-MT10 from Kaohsiung Medical University hospital, and KMTTH-107-028, KMTTH-108-011, KMTTH-109-037 from Kaohsiung Municipal Ta-tung Hospital.

\section{Data Availability Statement}

The data that support the findings of this study are available from the authors, YSF, CHC, THT, and CLL, upon reasonable request.

\section{References}

1. Zhang JN, Fan Y, Hao DJ. Risk factors for robot-assisted spinal pedicle screw malposition. Sci Rep. 2019;9(1):3025.

2. Devito DP, Kaplan L, Dietl R, Pfeiffer M, Horne D, Silberstein B, et al. Clinical acceptance and accuracy assessment of spinal implants guided with SpineAssist surgical robot: retrospective study. Spine (Phila Pa 1976). 2010;35(24):2109-15.

3. Ringel F, Stuer C, Reinke A, Preuss A, Behr M, Auer F, et al. Accuracy of robot-assisted placement of lumbar and sacral pedicle screws: a prospective randomized comparison to conventional freehand screw implantation. Spine (Phila Pa 1976). 2012;37(8):E496-501.

4. Onen MR, Simsek M, Naderi S. Robotic spine surgery: a preliminary report. Turk Neurosurg. 2014;24(4):512-8.

5. Hu X, Ohnmeiss DD, Lieberman IH. Robotic-assisted pedicle screw placement: lessons learned from the first 102 patients. Eur Spine J. 2013;22(3):661-6.

6. Hu X, Lieberman IH. What is the learning curve for robotic-assisted pedicle screw placement in spine surgery? Clin Orthop Relat Res. 2014;472(6):1839-44.

7. Macke JJ, Woo R, Varich L. Accuracy of robot-assisted pedicle screw placement for adolescent idiopathic scoliosis in the pediatric population. J Robot Surg. 2016;10(2):145-50.

8. Urakov TM, Chang KH, Burks SS, Wang MY. Initial academic experience and learning curve with robotic spine instrumentation. Neurosurg Focus. 2017;42(5):E4.

9. Hyun SJ, Kim KJ, Jahng TA, Kim HJ. Minimally Invasive Robotic Versus Open Fluoroscopic-guided Spinal Instrumented Fusions: A Randomized Controlled Trial. Spine (Phila Pa 1976). 2017;42(6):3538.

10. Schatlo B, Martinez R, Alaid A, von Eckardstein K, Akhavan-Sigari R, Hahn A, et al. Unskilled unawareness and the learning curve in robotic spine surgery. Acta Neurochir (Wien). 2015;157(10):1819-23; discussion 23. 
11. Kim HJ, Kang KT, Park SC, Kwon OH, Son J, Chang BS, et al. Biomechanical advantages of robotassisted pedicle screw fixation in posterior lumbar interbody fusion compared with freehand technique in a prospective randomized controlled trial-perspective for patient-specific finite element analysis. Spine J. 2017;17(5):671-80.

12. Cunningham S, Chellali A, Jaffre I, Classe J, Cao CGL. Effects of Experience and Workplace Culture in Human-Robot Team Interaction in Robotic Surgery: A Case Study. International Journal of Social Robotics. 2012;5(1):75-88.

13. Kuo KL, Su YF, Wu CH, Tsai CY, Chang CH, Lin CL, et al. Assessing the Intraoperative Accuracy of Pedicle Screw Placement by Using a Bone-Mounted Miniature Robot System through Secondary Registration. PLoS One. 2016;11(4):e0153235.

14. Tsai TH, Tzou RD, Su YF, Wu CH, Tsai CY, Lin CL. Pedicle screw placement accuracy of bone-mounted miniature robot system. Medicine (Baltimore). 2017;96(3):e5835.

15. Anderson JR. Cognitive skills and their acquisition. Hillsdale, N.J.: L. Erlbaum Associates; 1981. xiii, 386 p. p.

16. Donner Y, Hardy JL. Piecewise power laws in individual learning curves. Psychon Bull Rev. 2015;22(5):1308-19.

17. Joseph JR, Smith BW, Liu X, Park P. Current applications of robotics in spine surgery: a systematic review of the literature. Neurosurg Focus. 2017;42(5):E2.

18. Hackman JR. Groups That Work (and Those That Don't): Creating Conditions for Effective Teamwork. San Francisco: Jossey-Bass; 1990. 544 p.

19. Ilgen DR, Hollenbeck JR, Johnson M, Jundt D. Teams in organizations: from input-process-output models to IMOI models. Annu Rev Psychol. 2005;56:517-43.

20. Bajcsy R, Aloimonos Y, Tsotsos JK. Revisiting active perception. Auton Robots. 2018;42(2):177-96.

21. Kim HJ, Jung WI, Chang BS, Lee CK, Kang KT, Yeom JS. A prospective, randomized, controlled trial of robot-assisted vs freehand pedicle screw fixation in spine surgery. Int J Med Robot. 2017;13(3).

\section{Tables}

Table 1. Characteristics of patients in three groups 


\begin{tabular}{|c|c|c|c|c|}
\hline & $\begin{array}{l}\text { NS early-50 } \\
(2013-2014)\end{array}$ & $\begin{array}{l}\text { Ortho early-50 } \\
\text { (2014-2015) }\end{array}$ & $\begin{array}{l}\text { NS later-50 } \\
(2014-2015)\end{array}$ & $P$ value \\
\hline Case No. & 50 & 50 & 50 & \\
\hline Age (yrs) & & & & $0.4677 * \star \star *$ \\
\hline mean $\pm S D$ & $64.1 \pm 16.4$ & $61.3 \pm 18.4$ & $65.0 \pm 9.4$ & \\
\hline Sex & & & & $0.1451^{\star \star}$ \\
\hline Male/Female & $17 / 33$ & $20 / 30$ & $11 / 39$ & \\
\hline $\mathrm{BMI}\left(\mathrm{kg} / \mathrm{m}^{2}\right)$ & & & & $0.4703^{\star \star *}$ \\
\hline mean $\pm S D$ & $25.1 \pm 3.1$ & $24.8 \pm 3.4$ & $25.8 \pm 5.0$ & \\
\hline Segments & & & & $<0.0001^{* *}$ \\
\hline 2 levels ( $\leqq 4$ screws) & 30 & 8 & 38 & \\
\hline 3 levels (6 screws) & 13 & 23 & 11 & \\
\hline$>3$ levels (>6screws) & 7 & 19 & 1 & \\
\hline No. of Screws & 254 & 363 & 224 & \\
\hline Time/screw (min) & & & & $0.0017^{*}$ \\
\hline mean $\pm S D$ & $9.56 \pm 4.19$ & $7.29 \pm 3.64$ & $8.74 \pm 5.77$ & \\
\hline \multicolumn{5}{|l|}{ Accuracy } \\
\hline \multirow[t]{2}{*}{ Overall screws } & $99.6 \%(253 / 254)$ & $99.5 \%$ & $99.1 \%(222 / 224)$ & \\
\hline & & $(361 / 363)$ & & \\
\hline By cases: & & & & $0.7735^{\star \star}$ \\
\hline $100 \%$ & 49 & 48 & 49 & \\
\hline$<100 \%$ & 1 & 2 & 1 & \\
\hline
\end{tabular}

NS: Neurosurgery; Ortho: Orthopedic surgery; *: test by Wilcoxon sign rank test; **: test by Chi-squared test; ***: test by ANOVA; SD: standard deviation; BMI: body mass index.

\section{Table 2. Time per screw and learning curve between 5 doctors}

SD: standard deviation; SE: standard error; *: test by Wilcoxon sign rank test; $* *$ : test by Parallelism F test. 


\begin{tabular}{llllll}
$\begin{array}{l}\text { Surgeons/years of } \\
\text { practice }\end{array}$ & $\begin{array}{l}\text { Year of Robotic spine } \\
\text { surgery }\end{array}$ & $\begin{array}{l}\text { Case } \\
\text { No. }\end{array}$ & Screws & $\begin{array}{l}\text { Time/screw } \\
(\mathrm{min}) \\
\text { mean } \pm \text { SD }\end{array}$ & $\begin{array}{l}\text { Growth rate } \\
\text { estimate } \pm \text { SE }\end{array}$ \\
\hline Dr. A/26 yrs & May, 2013-Jun, 2013 & 10 & 40 & $12.28 \pm 5.21^{*}$ & $-0.13 \pm 0.17^{\star *}$ \\
\hline Dr. B/22 yrs & Sep, 2013-Nov, 2014 & 10 & 40 & $6.38 \pm 1.54$ & $0.08 \pm 0.92$ \\
\hline Dr. C/15 yrs & Sep, 2014-Jun, 2015 & 10 & 40 & $8.68 \pm 3.10$ & $-1.41 \pm 0.0$ \\
\hline Dr. D/11 yrs & Apr, 2014-Jun, 2017 & 10 & 40 & $6.33 \pm 1.90$ & $-0.57 \pm 1.6$ \\
\hline Dr. E/22 yrs & Aug, 2014-Jun, 2016 & 10 & 40 & $6.73 \pm 1.81$ & $0.75 \pm 0.0$ \\
\hline & & p-value & & $0.001 *$ & $<0.0001 * *$
\end{tabular}

\section{Figures}

\section{Timeline of Robotics at KMUH}

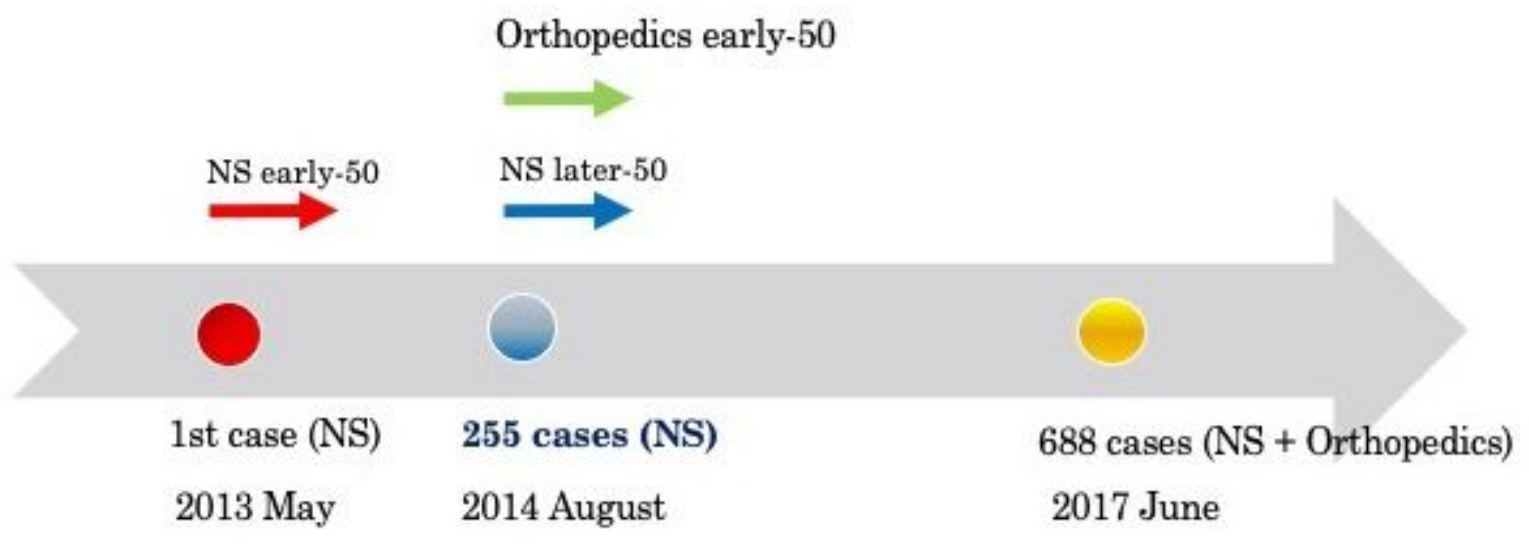

Figure 1

Timeline of robotic spine surgery at Kaohsiung Medical University Hospital (KMUH). The orthopedic surgeons joined the robotic spine surgery team in August 2014. At that time, 255 cases and 1237 screws had been placed successfully. (NS: cases performed by neurosurgeons). 
(A)

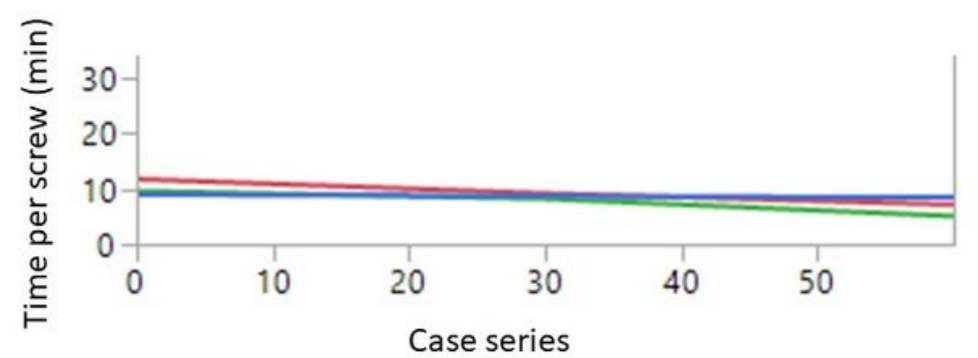

(B)

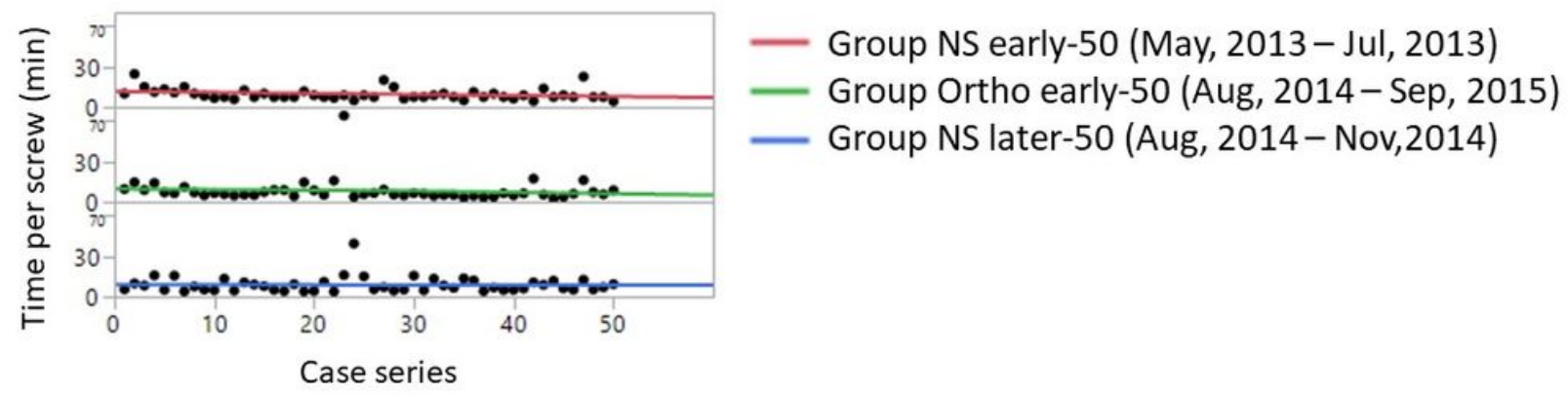

\section{Figure 2}

Groups learning curves The learning curve of the 3 groups and the test of parallelism were plotted. The upper part $(A)$ is the curves of the 3 groups put combined. The lower part of the figure (B) is the curve for each group. The parallelism of these curves was tested using the Parallelism $\mathrm{F}$ test and showed no significance. 


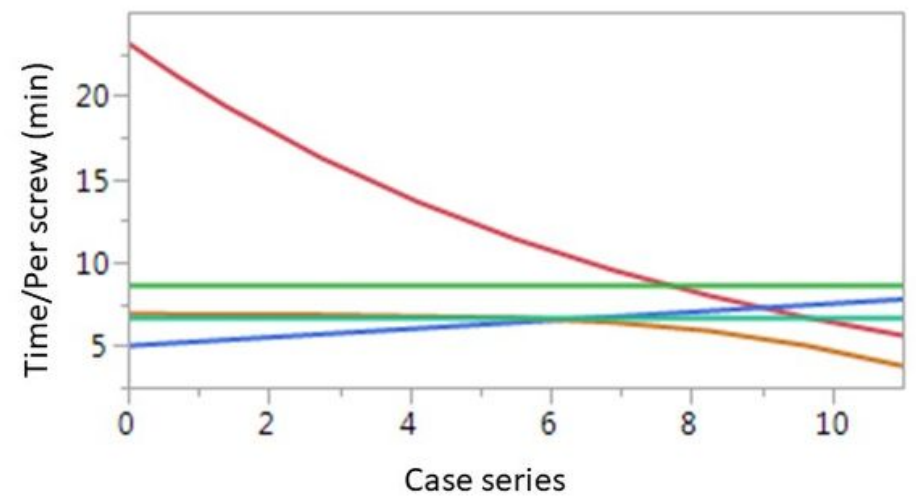

Dr. A: May, 2013- Jun, 2013

Dr. B: Sep, 2013 - Nov,2014

Dr. C: Sep, 2014 - Jun, 2015

Dr. D: Apr, 2014 - Jun, 2017

Dr. E: Aug, 2014 - Jun, 2016

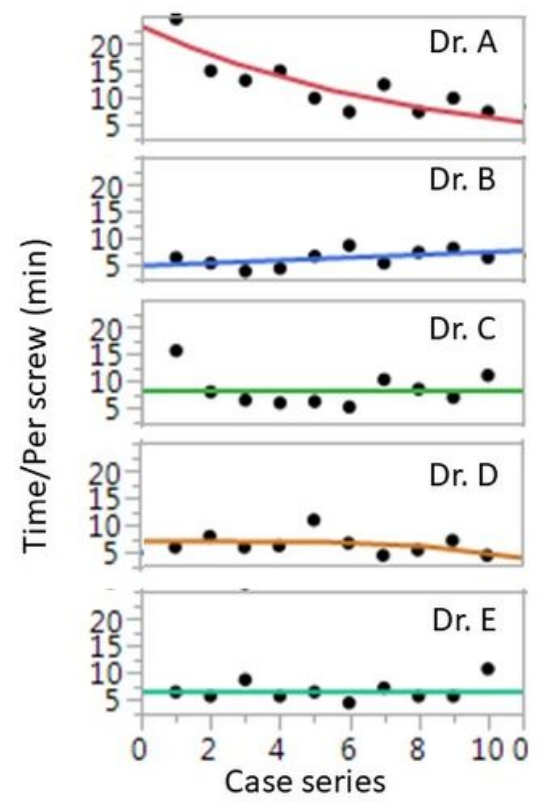

\section{Figure 3}

Individual surgeon's learning curve The learning curves of individual surgeons and the test of parallelism were plotted. The right side of the figure is the curve for each surgeon. The left side is the curves of the 5 surgeons combined. The parallelism of these curves, tested using the Parallelism F test, showed a significant difference among the curves. 